\title{
Multifrequency Astrophysics: An Updated Review
}

\author{
Franco Giovannelli*i \\ INAF - Istituto di Astrofisica e Planetologia Spaziali, Via del Fosso del Cavaliere, 100, 00133 \\ Roma, Italy \\ E-mail: Eranco.giovannellidiaps.inaf.it \\ Lola Sabau-Graziati \\ INTA-Dpt. Cargas Utiles y Ciencias del Espacio, C/ra de Ajalvir, Km 4 - E28850 Torrejón de \\ Ardoz, Madrid, Spain \\ E-mail: sabaumdeinta.es
}

In this paper - a short updated version of our review paper about "The impact of space experiments on our knowledge of the physics of the Universe (Giovannelli \& Sabau-Graziati, 2004) (GSG2004) and subsequent updating (Giovannelli \& Sabau-Graziati, 2012a, 2014a) - we will briefly discuss old and new results obtained in astrophysics, that marked substantially the research in this field. Thanks to the results, chosen by us following our knowledge and feelings, we will go along different stages of the evolution of our Universe discussing briefly several examples of results that are the pillars carrying the Bridge between the Big Bang and Biology.

We will remark the importance of the joint venture of 'active physics experiments' and 'passive physics experiments' ground- and space-based either big either small in size that, with their results, are directed towards the knowledge of the physics of our universe. New generation experiments open up new prospects for improving our knowledge of the aforementioned main pillars.

XI Multifrequency Behaviour of High Energy Cosmic Sources Workshop

25-30 May 2015

Palermo, Italy

*Speaker.

${ }^{\dagger}$ A footnote may follow. 


\section{Introduction}

The birth of the universe and its present status constitute the two banks of a river in which the life of the universe is slowly flowing. Undoubtedly the two banks are joined by a bridge that Giovannelli (2001a) nicknamed "The Bridge between the Big Bang and Biology" that constituted the title of the workshop held in Stromboli (Aeolian Archipelago, Sicily, Italy) in 1999.

In this paper we will briefly discuss the main pillars of this bridge by using the huge amount of experimental data coming from "Active Physics Experiments (APEs)" and from "Passive Physics Experiments (PPEs)" ground- and space-based. The APEs try to reproduce in laboratory the physical conditions of our Universe at the beginning of its life and later, while the PPEs try to observe our Universe after the epoch of recombination, when the Cosmic Microwave Background (CMB) gives witness of the conditions of the primeval Universe, and later - after the epoch of reionization, when the first stars appear - for providing information about the formation of galaxies, "active" and "normal", quasars (QSOs), and all the processes giving rise to Gamma Ray Bursts (GRBs), stellar evolution and Supernovae explosions. These latter phenomena are responsible of the injection of heavy elements in the interstellar medium, condition necessary for the formation of rocky planets, and then maybe the flowering of the life.

For describing the origin of our Universe, the Big Bang model (BBM) is generally accepted, but it is not complete. Indeed, the BBM is based on the Cosmological Principle which assumes that matter in the universe is uniformly distributed on all scales. This is a very useful approximation that allows one to develop the basic Big Bang scenario, but a more complete understanding of our Universe requires going beyond the Cosmological Principle. Many cosmologists suspect that the inflation theory may provide the framework for explaining the large-scale uniformity of our universe and the origin of structure within it.

Spergel et al. (2003) by using the first year data from WMAP derived for the age of the Universe $13.7 \pm 0.2 \mathrm{Gyr}$. The WMAP determination of the age of the universe implies that globular clusters form within $2 \mathrm{Gyr}$ after the Big Bang, a reasonable estimate that is consistent with structure formation in the $\Lambda \mathrm{CDM}$ cosmology. Recent measurements from WMAP provide an age of our Universe of $(13.77 \pm 0.059) \times 10^{9} \mathrm{yr}$ (Komatsu \& Bennett, 2014).

Before to go along the different stages of the evolution of our Universe crossing the Bridge between the Big Bang and Biology we need to remark a fundamental antecedent: nuclear reactions in stars.

\section{Nuclear Reactions in Stars}

If we have not experimental information about the cross sections of nuclear reactions occurring in the stars it is hard to describe the correct star evolution.

The knowledge of the cross-sections of nuclear reactions occurring in the stars appears as one of the most crucial points of all astroparticle physics. Direct measurements of the cross sections of the ${ }^{3} \mathrm{He}\left({ }^{4} \mathrm{He}, \gamma\right){ }^{7} \mathrm{Be}$ and ${ }^{7} \mathrm{Be}(\mathrm{p}, \gamma){ }^{8} \mathrm{Be}$ reactions of the $p p$ chain and ${ }^{14} \mathrm{~N}(\mathrm{p}, \gamma){ }^{15} \mathrm{O}$ reaction of the CNO-cycle will allow a substantial improvement in our knowledge on stellar evolution. 
The LUNA (Laboratory for Underground Nuclear Astrophysics) is devoted to measure nuclear cross sections relevant in astroparticle physics. It is the most valuable experiment running underground in the Gran Sasso Laboratory of the INFN.

The LUNA collaboration has already measured with good accuracy the key reactions $\mathrm{D}(\mathrm{p}, \gamma)^{3} \mathrm{He}$, ${ }^{3} \mathrm{He}(\mathrm{D}, \mathrm{p}){ }^{4} \mathrm{He}$ and ${ }^{3} \mathrm{He}\left({ }^{4} \mathrm{He}, \gamma\right){ }^{7} \mathrm{Be}$. These measurements substantially reduces the theoretical uncertainty of $\mathrm{D},{ }^{3} \mathrm{He},{ }^{7} \mathrm{Li}$ abundances. The $\mathrm{D}\left({ }^{4} \mathrm{He}, \gamma\right){ }^{6} \mathrm{Li}$ cross section - which is the key reaction for the determination of the primordial abundance of ${ }^{6} \mathrm{Li}$ - has been measured (e.g. Gustavino, 2007, 2009, 2011, 2012, 2013), as well as that of ${ }^{2} \mathrm{H}(\alpha, \gamma){ }^{6} \mathrm{Li}$ (Anders et al., 2013), and ${ }^{2} \mathrm{H}(\alpha, \gamma){ }^{6} \mathrm{Li}$ (Anders et al., 2014).

Other reactions fundamental for a better knowledge of stellar evolution have been studied by the LUNA experiment: e.g. ${ }^{17} \mathrm{O}(\mathrm{p}, \gamma){ }^{18} \mathrm{~F}$ (Scott et al. 2012); ${ }^{25} \mathrm{Mg}(\mathrm{p}, \gamma){ }^{26} \mathrm{Al}$ (Strieder et al., 2012) ${ }^{25} \mathrm{Mg}(\mathrm{p}, \gamma){ }^{26} \mathrm{Al}$ (Straniero et al., 2013); ${ }^{17} \mathrm{O}(\mathrm{p}, \gamma){ }^{18} \mathrm{~F}$ (Di Leva et al., 2014).

A general data base for Experimental Nuclear Reaction Data (EXFOR) can be found in: https://www-nds.iaea.org/exfor/exfor.htm.

\section{A Swift Journey along the Bridge between the Big Bang and Biology}

Because of the limited length of this paper we will briefly discuss several old and new exciting results that can be considered, in accordance with our opinion, the most important pillars supporting the Bridge between the Big Bang and Biology.

\subsection{Big Bang and Standard Model}

Undoubtedly the advent of new generation experiments ground-and space-based have given a strong impulse for verifying current theories, and for providing new experimental inputs for developing a new physics for going, probably, over the standard model (SM). Recent results coming from Active Physics Experiments (APEs) and Passive Physics Experiments (PPEs) have opened such a new path.

The hunt to Higgs boson - often called "the God particle" because it's said to be what caused the "Big Bang" that created our Universe: matter obtains mass interacting with Higgs field - started a few years ago with the most powerful accelerators constructed in the world, in particular with the different experiments of the Large Hadron Collider (LHC). These experiments can provide information about the first moment of the life of the Universe. LHC is a complementary tool for HE observatories looking directly to the Universe. From ATLAS results, a $5.0 \sigma$ excess at $\sim 126.5 \mathrm{GeV}$ has been detected. This value is compatible with the expected mass of Higg's boson (Gianotti, 2012). The Compact Muon Solenoid (CMS) experiment at LHC detected a new boson at $125.3 \pm 0.6 \mathrm{GeV}$ with $4.9 \sigma$ significance (Incandela, 2012). This result, together with that from ATLAS, if confirmed, would complete the SM of physics.

Thanks to collisions at $13 \mathrm{TeV}$ the experiment Large Hadron Collider beauty (LHCb) at LHC detected a new particle: the Pentaquark. The existence of the pentaquark was theoretically suggested since 1960-ies (Gell-Mann, 1964). Pentaquark gives a new way for the combination of the quarks that are the fundamental constituents of neutrons and protons (Cardini, 2015; Aaij et al., 2015). 
Recently the collaboration of the BCEP2 experiment claims the detection of E-mode (Crites et al., 2015) and B-mode polarization of the Cosmic Microwave Background at at $7.0 \sigma$ significance (CMB) (Ade et al., 2015). If B-mode polarization would be confirmed, the inflationary model of the Universe would be definitively confirmed. However, big discoveries need big confirmations.
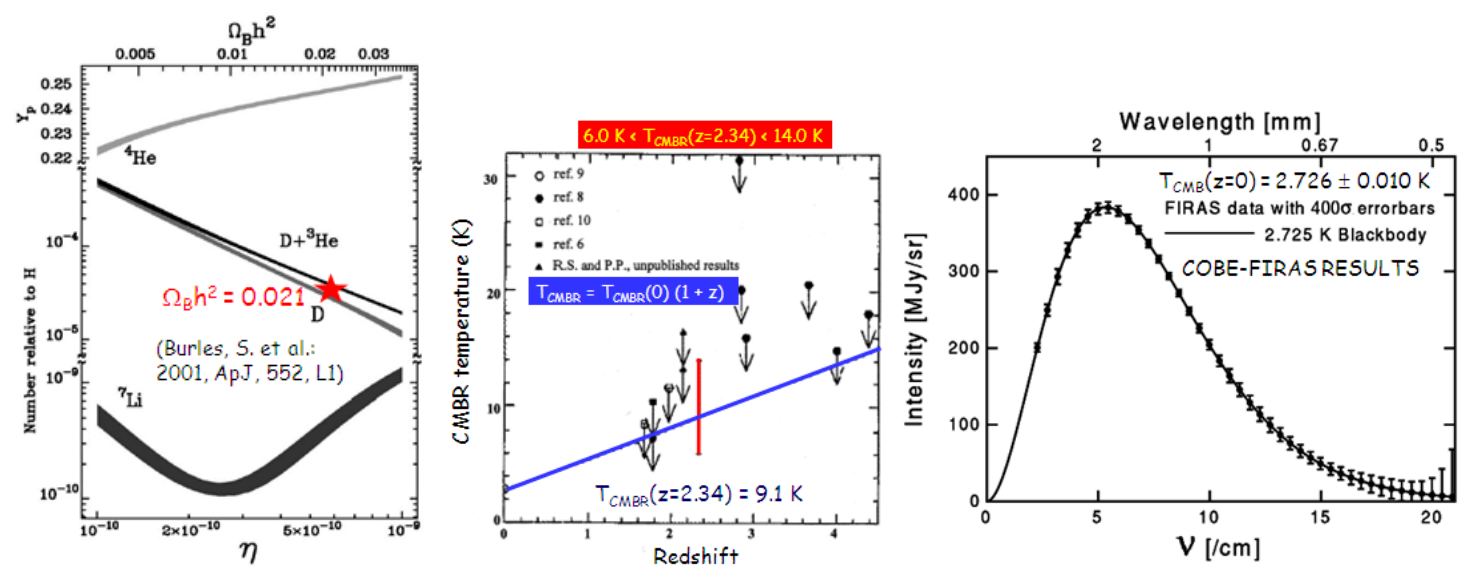

Figure 1: Three experimental results in favor of the BBM (see text for explanation).

However, in the last decade several experiments provided results confirming the validity of the BBM. In Fig. 1 one can see: i) (left panel): red star - the experimental confirmation of the content of the primordial light elements (de Bernardis et al., 2000) superimposed to the theoretical curves (Burles et al., 2001); ii) (middle panel): red line - the temperature of the Cosmic Microwave Background Radiation $\left(\mathrm{T}_{\mathrm{CMBR}}\right.$ ) at redshift $\mathrm{z}=2.34$, ranging between 6 and $14 \mathrm{~K}$ (Srianand, Petitjean \& Ledoux, 2000), in agreement with the theoretical temperature law $\mathrm{T}_{\mathrm{CMBR}}=\mathrm{T}_{\mathrm{CMBR}}(0)(1+\mathrm{z})$, which gives at $\mathrm{z}=2.34$ a temperature of $9 \mathrm{~K}$; iii) (right panel): the $\mathrm{CMB}$ radiation temperature $(2.726 \pm 0.010 \mathrm{~K})$ (Bartelmann, 2008, after Mather et al., 1990).

\subsection{Background Radiation in the Universe}

The Diffuse Extragalactic Background RAdiation (DEBRA) permeates through the whole electromagnetic spectrum, and it is peaked in the microwave region. It is possible to consider the DEBRA as a radiation produced by a cosmic source: the whole Universe. Such a background radiation from radio to HE $\gamma$-ray energy bands has been deeply discussed by Ressell \& Turner (1990), and in GSG2004 and the references therein. The analysis of the different components of DEBRA leads to the Grand Unified Photon Spectrum (GUPS), covering 29 orders of magnitude of the electromagnetic spectrum, from $10^{-9}$ to $10^{20} \mathrm{eV}$, as shown in Fig. 2 (after Ressell \& Turner, 1990). The light-red and the light-indico rectangles indicate the domains with energies less or greater than $\approx 10 \mathrm{GeV}$, respectively. The domain at higher energies is now explored by numerous experiments space-based, like Fermi LAT observatory (up to $300 \mathrm{GeV}$ ) and ground-based, like Whipple, Veritas, HESS, Magic, and the coming CTA (Cherenkov Telescopes Array). All these experiments will provide to fill the zone of the GUPS diagram prepared by Ressell \& Turner (1990) where only upper limits were reported.

Henry $(1999,2002)$ thoroughly discussed the updated experimental situation of the cosmic background. 


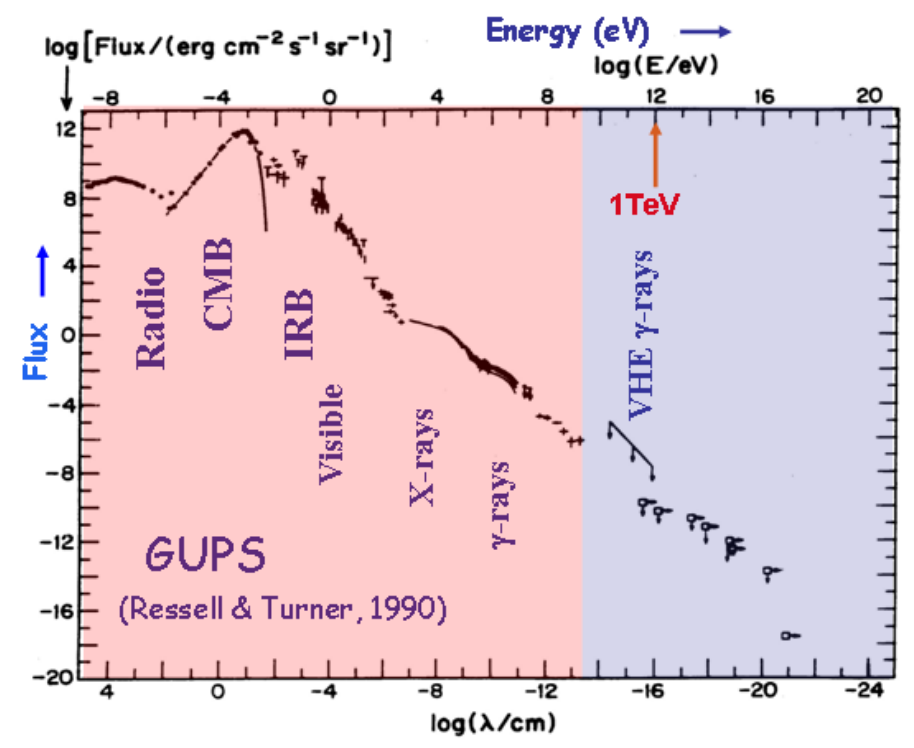

Figure 2: The Grand Unified Photon Spectrum of the Diffuse Extragalactic Background Radiation (after Ressell \& Turner, 1990).

\subsection{Extragalactic Background Light}

The intergalactic space is filled with the light produced by all the stars and accreting compact objects that populated the observable Universe throughout the whole cosmic history. This relic cosmic background from IR to UV is called the diffuse Extragalactic Background Light (EBL).

Figure 3 shows: (upper panel) - the contribution to EBL of redshifted dust emission and redshifted star light (after models by Meyer et al., 2012; Inoue et al., 2013); (lower panel) - experimental data clearly reproducing the shape of the former contributions (after Krennrich \& Orr, 2013; Beatty et al., 2013). The light-red and light-indico rectangles show the zones of redshifted dust emission and redshifted star light, respectively.

Direct measurements of the EBL are difficult due to bright local foregrounds. A powerful approach for probing these diffuse radiation fields in the UV to far-IR bands is through $\gamma-\gamma$ absorption of high-energy photons. Actually pair production $\left(\mathrm{e}^{+} \mathrm{e}^{-}\right)$against EBL photons with wavelengths from ultraviolet to infrared is effective at attenuating $\gamma$-rays with energy above $\sim 10 \mathrm{GeV}$. This process introduces an attenuation in the spectra of $\gamma$-ray sources above a critical energy (e.g. Costamante, 2012; Buson, 2014).

The last decade has been foreboding of a full coverage of the HE-VHE $\gamma$-ray energy band, thanks to the many ground- and space-based high sensitivity experiments, as shown in Fig. 4. These experiments have provided a large amount of data from many extragalactic emitters at high redshift (e.g. Costamante, 2012). Thanks to measurements of the quasar 3C 279 ( $\mathrm{z} \simeq 0.54$ ) obtained with the MAGIC experiment (Albert et al., 2008), and with the many sources at high redshift, including Gamma Ray Bursts (GRBs) measured with the FERMI observatory (Abdo et al., 2010), it has been demonstrated that the Universe is more transparent to $\gamma$-rays than before believed (Coppi \& Aharonian, 1997). 

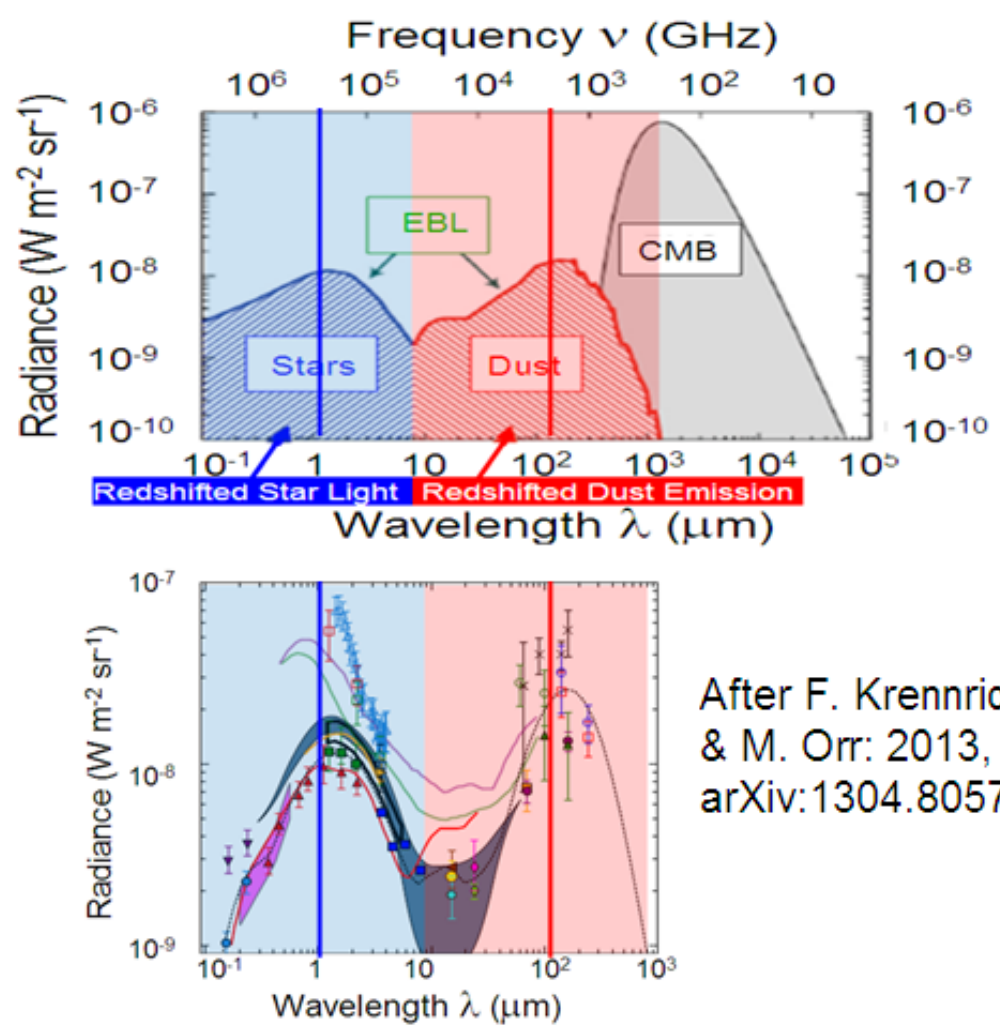

After F. Krennrich

\& M. Orr: 2013, arXiv:1304.8057

Figure 3: Extragalactic Background Light (EBL): upper panel - the contribution to EBL of redshifted dust emission and redshifted star light (after models by Meyer et al., 2012; Inoue et al., 2013); lower panel experimental data clearly reproducing the shape of the former contributions (after Krennrich \& Orr, 2013). For a comparison of the two plots, red and blue vertical lines mark 100 and $1 \mu \mathrm{m}$ wavelength, respectively.

\subsection{Is the Universe Flat?}

One of the most critical points about our Universe is the problem of its flatness. The present state of the cosmological tests is illustrated in Fig. 5. The left panel of Fig. 5 shows the results obtained with the BOOMERanG (Balloon Observations Of Millimetric Extragalactic Radiation and Geomagnetics) experiment (de Bernardis et al., 2000). They are fully consistent with a spatially flat Universe. The right panel of Fig. 4 shows the combination of the likelihood contours obtained with three different observational approaches: i) type-Ia SNe (Tonry et al., 2003; Riess et al. 2004); ii) CMB (Spergel et al. 2003; Bennett et al. 2013); iii) galaxy clusters (Schuecker et al. 2003; Schuecker, 2005). One can see that the cosmic matter density is close to $\Omega_{m}=0.3$, and that the normalized cosmological constant is around $\Omega_{\Lambda}=0.7$. This sums up to unit total cosmic energy density and suggests a spatially flat universe. However, the density of cosmic matter growths with redshift like $(1+z)^{3}$ whereas the density $\rho_{\Lambda}$ related to the cosmological constant $\Lambda$ is independent of z. The final results from WMAP (Bennett et al., 2013) shows a little misalignment with the line of "flat Universe". Thus it is necessary to be careful in the conclusions.

\subsection{Hubble Constant}

The Hubble constant $\left(\mathrm{H}_{0}\right)$ is one of the most important numbers in cosmology because it is 


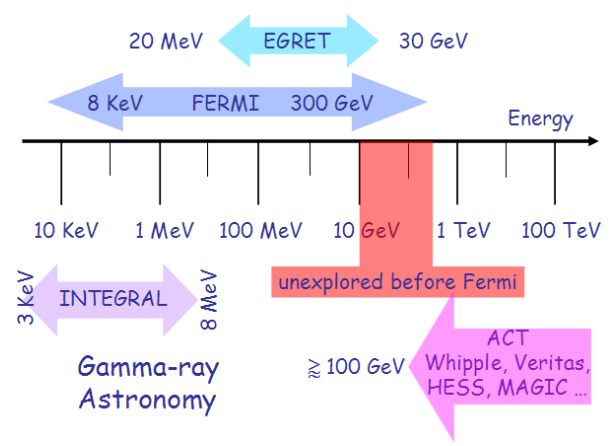

Figure 4: The HE-VHE $\gamma$-ray energy band completely explored with the new generation ground- and space-based experiments.

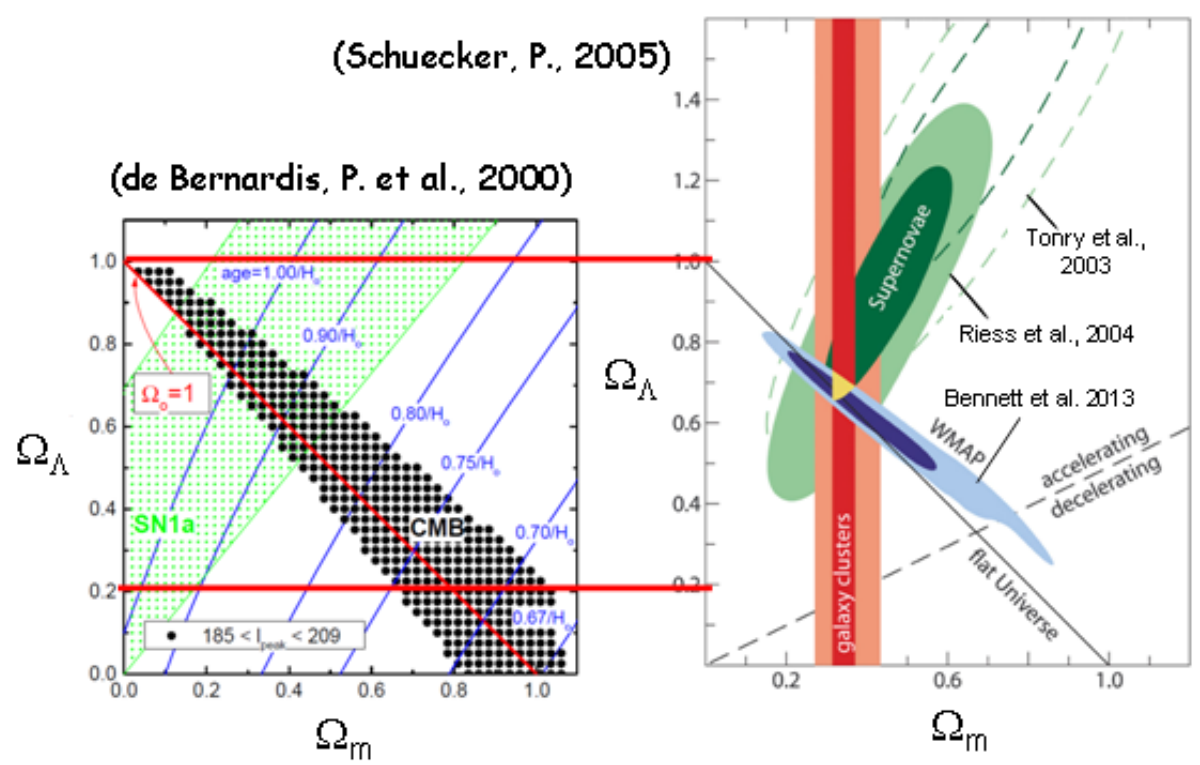

Figure 5: Constraints of cosmological parameters (after de Bernardis et al., 2000; Schuecker, 2005, Bennett et al., 2013).

needed to estimate the size and age of the universe. The important problem of determination of $\mathrm{H}_{0}$ value is one of the most exciting. Indeed, in the literature it is possible to find many determinations coming from different experiments using different methods. However, it is very complicate to obtain a true value for $\mathrm{H}_{0}$. It is necessary to have two measurements: i) spectroscopic observations that reveal the galaxy's redshift, indicating its radial velocity; ii) the galaxy's precise distance from Earth (and this is the most difficult value to determine).

A large summary about the methods used for $\mathrm{H}_{0}$ determination, and its derived values can be found in the Proceedings of the Fall 2004 Astronomy 233 Symposium on "Measurements of the Hubble constant" (Damon et al., 2004). A recent discussion about the Hubble constant has been published by Giovannelli \& Sabau-Graziati (2014a), where it is possible to find also a large number of references, reporting the many controversial evaluations of $\mathrm{H}_{0}$.

However, Riess et al. (2011) with the HST determined a value of $\mathrm{H}_{0}=73.8 \pm 2.4 \mathrm{~km} \mathrm{~s}^{-1}$ 
$\mathrm{Mpc}^{-1}$. This value agrees with the WMAP results: $\mathrm{H}_{0}=71.0 \pm 2.5 \mathrm{~km} \mathrm{~s}^{-1} \mathrm{Mpc}^{-1}$ (Komatsu et al., 2011). Bennett et al. (2014) discussed the progress occurred in recent years for determining the Hubble constant: results coming from the cosmic distance ladder measurements at low redshift and $\mathrm{CMB}$ measurements at high redshift. The $\mathrm{CMB}$ is used to predict the current expansion rate of the universe by best-fitting cosmological model. At low redshift baryon acoustic oscillation (BAO) measurements have been used - although they cannot independently determine $\mathrm{H}_{0}$ - for constraining possible solutions and checks on cosmic consistency. Comparing these measurements they found $\mathrm{H}_{0}=69.6 \pm 0.7 \mathrm{~km} \mathrm{~s}^{-1} \mathrm{Mpc}^{-1}$.

Does this determination, finally, close the history about the search of the "true" value of $\mathrm{H}_{0}$ ?

\subsection{Reionization Epoch}

Ground-based observations of the CMB on subdegree angular scales suggest that the gas content of the universe was mostly neutral since recombination at $\mathrm{z} \sim 1000$ until about $\mathrm{z} \sim 100$ (Gnedin, 2000 and the references therein) because earlier reionization would have brought the last scattering surface to lower redshift, smoothing the intrinsic CMB anisotropy. At the same time, we know that the universe is highly ionized, since $\mathrm{z} \approx 5$, from observations of the spectra of quasars with the highest redshifts (e.g. Giallongo et al. 1994). This change of the ionization state of the universe from neutral to highly ionized is called "reionization". How large is the redshift to which the reionization started and stopped is object of strong debate.

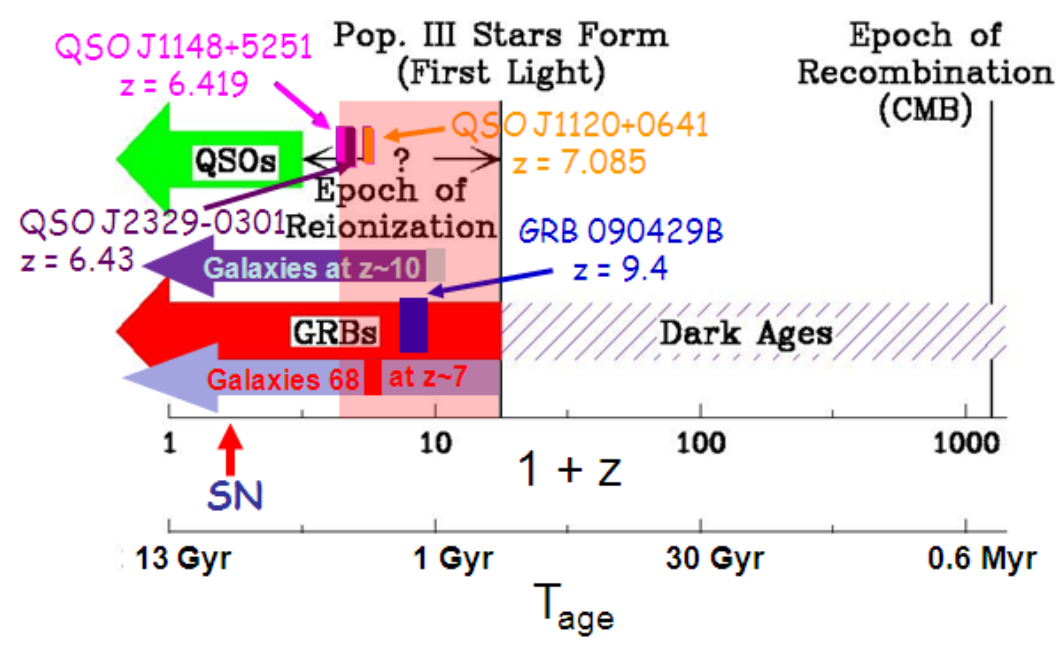

Figure 6: A sketch of reionization epoch (after Xiangping Wu's Talk at the Summer School on "Cosmic Reionization" at the KIAA-PKU , Beijing, China, July 1-11, 2008).

The formation of the first stars and quasars marks the transformation of the universe from its smooth initial state to its clumpy current state. In current cosmological models, the first sources of light began to form at a redshift $\mathrm{z} \sim 30$ and reionized most of the hydrogen in the universe by $\mathrm{z} \sim 7$ (see review by Loeb \& Barkana, 2001).

The argument for an extended period of reionization is now proved by measurements. Indeed, the WMAP has detected the correlation between temperature and polarization on large angular scales (Kogut et al., 2003) that has an amplitude proportional to the total optical depth of CMB 
photons to Thomson scattering, $\tau$ (Kaplinghat et al., 2003; Sunyaev \& Zeldovich, 1980; Zaldarriaga, 1997).

Modeling reionization with a single sharp transition at $\mathrm{z}_{\mathrm{ri}}$, a multi-parameter fit to the WMAP data gives $z_{\mathrm{ri}}=17 \pm 5$ (Spergel et al., 2003). On the other hand, the evolution of quasar spectra from $\mathrm{z} \approx 7$ and $\mathrm{z} \approx 6$ shows a rapid decrease in the amount of neutral Hydrogen, indicating the end of reionization (Fan et al., 2003). A simple interpretation to explain these two very different datasets is that reionization started early, $\mathrm{z}_{\mathrm{ri}} \sim 20$, but did not conclude until much later $(\mathrm{z} \sim 6$ ) (Knox, 2003).

The WMAP detection of reionization (Kogut et al. 2003) implies the existence of an early generation of stars able to reionize the universe at $\mathrm{z} \sim 20$. Panagia et al. (2005) in deep HST/VLT/Spitzer images found that the source UDF 033238.7-274839.8 - a post-starburst galaxy with a mass $\sim 6 \times 10^{11} \mathrm{M}_{\odot}$ placed at $\mathrm{z} \geq 6.5$ - may be capable of reionizing its surrounding region of the universe, starting the process at a redshift as high as $\mathrm{z}=15 \pm 5$.

Figure 6 shows schematically the updated experimental situation about cosmic sources (galaxies, GRBs, QSOs, SNe) detected at high redshifts. The light-red rectangle marks the possible range of $\mathrm{z}$ during which the reionization occurred.

However, although there is rather good agreement about the epoch of reionization, how really reionization occurs is still object of debate. Indeed, Dopita et al. (2011), considering that recent observations show that the measured rates of star formation in the early universe are insufficient to produce reionization, suggest the presence of another source of ionizing photons. This source could be the fast accretion shocks formed around the cores of the most massive haloes.

\subsection{A Unified Scheme for Collapsed Objects}

The argument of the possibility of describing all the collapsed objects with a unique scheme have been discussed since long time by many authors. In the review paper by Begelman, Blandford $\&$ Rees (1984) they discussed the theory of extragalactic radio sources and in particular the unified model of active galactic nuclei (AGNs).

From the evidence that the shapes of SEDs (Spectral Energy Distributions) of different kind of AGNs (Cen A, NGC 4151, and 3C 273) are practically the same (e.g. Ramaty \& Lingenfelter, 1982), Giovannelli \& Polcaro (1986) (GP86), by using experimental data coming from the EINSTEIN observatory, constructed the maximum luminosity diagram $\left(\mathrm{L}_{\mathrm{xmax}}(\mathrm{z})\right)$ for extragalactic objects, independent of the current classification of those objects. Indeed, those extragalactic objects have the same engine producing energy (supermassive black hole with accretion disk and jet) and they are classified as blazars, or radio-loud QSOs, or radio galaxies depending on the angle between the line of sight and the jet axis (e.g. Urry \& Padovani, 1995; Padovani, 1999). The attenuation in the emission of a cosmic source containing a black hole in function of such an angle and the beam Lorentz's factor of the particles have been calculated by Bednarek et al. (1990).

The numerical continuity of the whole $\mathrm{L}_{\mathrm{xmax}}(\mathrm{z})$ function should be interpreted as owed to an evolution of the central X-ray source from a very active to a more quiet status. Moreover, the part of GP86 at lower redshifts converge to the level of emission due to the discrete sources within the galaxies. This was recently supported by the results of Mineo et al. (2014) that provide a range of possible emission of discrete sources in the galaxies between $\approx 10^{39}$ and $\approx 10^{40} \mathrm{erg} \mathrm{s}^{-1} \mathrm{M}_{\odot}^{-1} \mathrm{yr}^{-1}$. 
For a more detailed discussion see the review papers by Giovannelli \& Sabau-Graziati (2004, 2014a).

\subsection{Jets in Astrophysics}

Every object rotating with adequate energy produces a jet. Relativistic jets have been found in numerous galactic and extragalactic cosmic sources at different energy bands. They can be formed by electrons and protons - accelerated up to relativistic energies - which through interactions with the matter and/or photons generate high energy radiation. The spectra of such a radiation are strongly dependent on the angle formed by the beam axis and the line of sight, and obviously by the Lorentz factor of the particles (e.g. Bednarek et al., 1990 and the references therein; Beall, Guillory \& Rose, 1999; Beall, 2002, 2003; Beall et al., 2006, 2007).

Jets are thought to be produced by the powerful electromagnetic forces created by magnetized gas swirling toward a collapsed object (i.e. black hole). Although most of the material falls into the collapsed object, some can be ejected at extremely high speeds. Magnetic fields spun out by these forces can extend over vast distances and may help explain the narrowness of the jet (e.g. Clarke et al., 2008).

However, highly collimated supersonic jets and less collimated outflows are observed to emerge from a wide variety of astrophysical objects. They are seen in young stellar objects (YSOs), protoplanetary nebulae, compact objects (like galactic black holes or microquasars, and X-ray binary stars), and in the nuclei of active galaxies (AGNs). Despite their different physical scales (in size, velocity, and amount of energy transported), they have strong morphological similarities. What physics do they share? These systems are either hydrodynamic or magnetohydrodynamic (MHD) in nature and are, as such, governed by non-linear equations. An important review on this topic was published by de Gouveia dal Pino (2005). Very interesting discussion has been published about the role of magnetic reconnection on jet/accretion disk systems, valid in different kind of cosmic sources, like from microquasars to low luminous AGNs, till YSOs (de Gouveia Dal Pino, Piovezan \& Kadowaki, 2010).

Astrophysical jets are a remarkable laboratory for a number of important physical processes. They provide a confirmation of special relativity in terms of relativistic Doppler boosting, superluminal motion, and time dilation effects. When coupled with their black-hole/neutron-star origins, jets have implications for testing general relativity. Over the course of two decades of astrophysical research, we have become aware that jets are ubiquitous phenomena in astrophysics. Extended linear structures now associated with jets can be found in star-forming regions, galactic binaries, microquasars, active galaxies and quasars, clusters of galaxies, and $\gamma$-ray bursts. The presence and evolution of these jet-like structures is of course a testament to the principle of conservation of angular momentum.

The association of jets with accretion disks strengthens the case for similar physical processes in all these phenomena (e.g., Beall, 2003; Marscher, 2005), and it has become plausible that essentially the same physics is working over a broad range of temporal, spatial, and luminosity scales. Jets have, therefore, become a 'laboratory' or perhaps an anvil, that we can use to help us forge our understanding of the physical processes in the sky.

In 1992 the first so-called microquasar, annihilateur, was identified (Mirabel et al., 1992). This source was exhibiting bipolar radio jets spread over several light-years. This was the first 


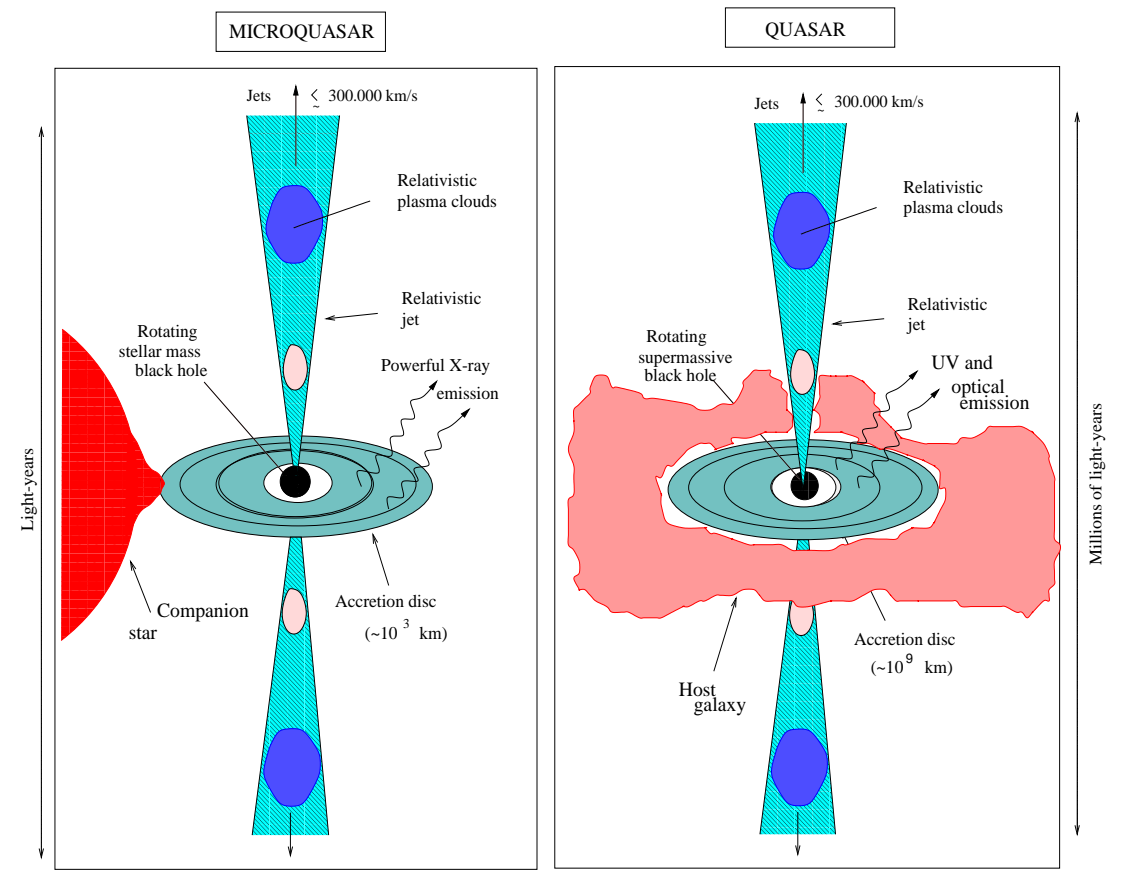

Figure 7: Sketch showing analogies between quasars and microquasars. Note the different mass and length scales between both types of objects (Chaty, 1998).

such observation in our Galaxy, however jets had been already observed emanating from distant galaxies. Therefore this observation made clear the existence of a morphological analogy between quasars and microquasars. Indeed, Mirabel \& Rodríguez (1994) detected from the black hole candidate GRS 1915+105 - discovered by Castro Tirado et al. (1994) - apparent superluminal motions, while frame velocity was $\mathrm{v} \sim 0.92 \mathrm{c}$. It became then rapidly clear that the advantages of microquasars compared to quasars were that i) they are closer, ii) it is possible to observe both (approaching and receding) jets, and iii) the accretion/ejection timescale is much shorter. After this observation of superluminal motions, the morphological analogy with quasars became stronger, and the question was then: is this morphological analogy really subtended by physics? If the answer is yes, then microquasars really are "micro"-quasars. For instance, there should exist microblazars (microquasar whose jet points towards the observer), in order to complete the analogy with quasars.

A schematic view of a microquasar, compared with quasars, is given in Figure 7 (Chaty, 1998).

Microquasars are among the best laboratories for high energy phenomena and astroparticle physics. They are good candidates to be emitters of astroparticles: very high energy photons, cosmic rays and neutrinos. For these reasons the study of microquasars is one of the main goal of current space missions. Since each component of the system emits at different wavelengths, it is necessary to undertake multifrequency observations in order to understand phenomena taking place in these objects.

Theoretical and observational works show that jets from AGN can trigger star formation. However, in the Milky Way the first - and so far - only clear case of relativistic jets inducing star formation has been found in the surroundings of the microquasar GRS 1915+105. Mirabel et al. (2015) discussed jet-induced star formation by a microquasar. Although star formation induced by 
microquasar jets may not be statistically significant in the Milky Way, jets from stellar black holes may have been important to trigger star formation during the re-ionization epoch of the universe (Mirabel et al. 2011).

A recent reviews about jets in astrophysics has been published by Beall (2014a; 2014b).

\subsection{Gamma Ray Bursts}

Gamma-ray burst (GRBs) were discovered in 1967 - thanks to the four VELA spacecrafts, originally designed for verifying whether the Soviet Union abided the 1963 Limited Nuclear Test Ban Treaty - when 16 strong events were detected (Klebesadel, Strong \& Olson, 1973). Since then GRBs have remained a puzzle for the community of high energy astrophysicists. For this reason the problem of GRBs originated thousands articles most of them devoted to their physical interpretation (e.g. the review by Mazets \& Golenetskii, 1988; the review by GSG2004 and the references therein). BATSE/CGRO experiment detected 2704 GRBs from 1991 to 1999. This number increased with new generation satellites (BeppoSAX, RossiXTE, HETE, INTEGRAL, SWIFT, and FERMI). From the BATSE and KONUS isotropic distribution of GRBs, their cosmological origin have been demonstrated. GRBs may be classified into TWO groups depending on their duration: $\sim 0.2 \mathrm{~s}(25 \%)$, and $\sim 30 \mathrm{~s} \mathrm{(75 \% )} \mathrm{(e.g.} \mathrm{Kouveliotou} \mathrm{et} \mathrm{al.,} \mathrm{1993).} \mathrm{The} \mathrm{counterparts} \mathrm{for} \mathrm{all} \mathrm{bursts}$ can be observed in all wavelengths (X, UV, opt, IR, radio): the afterglow (e.g. Kann et al., 2010; Perley et al., 2014).

Theoretical description of GRBs is still an open strongly controversial question as discussed elsewhere (e.g. Giovannelli \& Sabau-Graziati, 2008; Giovannelli, 2013). Many review papers have been published about GRBs. Among them we can cite those published in the last decade (Piran, 2004; Meszaros, 2006); Woosley \& Bloom, 2006; Granot, 2007, 2009; Granot \& Ramirez-Ruiz, 2010; Inoue et al., 2013). Recently an interesting review about short GRBs has been published by Berger (2014).

Important implications on the origin of the highest redshift GRBs are coming from the detection of the GRB 080913 at $\mathrm{z}=6.7$ (Greiner et al., 2009), GRB 090423 at $\mathrm{z} \sim 8.2$ (Tanvir et al., 2009), and GRB 090429B at $z=9.4$ (Cucchiara et al., 2011). This means that really we are approaching to the possibility of detecting GRBs at the end of Dark Era, where the first Pop III stars appeared. Izzo et al. (2010) discussed successfully a theoretical interpretation of the GRB 090423 within their fireshell model. Wang \& Dai (2009) studied the high-redshift star formation rate (SFR) up to $\mathrm{z} \simeq 8.3$ considering the Swift GRBs tracing the star formation history and the cosmic metallicity evolution in different background cosmological models including $\Lambda \mathrm{CDM}$, quintessence, quintessence with a time-varying equation of state and brane-world models. $\Lambda \mathrm{CDM}$ model is the preferred which is however compared with other results.

Although big progress has been obtained in the last few years, GRBs theory needs further investigation in the light of the experimental data coming from old and new satellites, often coordinated, such as BeppoSAX or BATSE/RXTE or ASM/RXTE or IPN or HETE or INTEGRAL or SWIFT or AGILE or FERMI or MAXI. Indeed, in spite of thousands papers appeared in the literature since the discovery of GRBs, the problem of their energy emission is still elusive: i) what is jet's composition? (kinetic or magnetic?); ii) where is dissipation occurring? (photosphere? deceleration radius?); iii) how is radiation generated? (synchrotron, Inverse Compton, hadronic?) (Zhang et al., 2013). 
For this reason we believe useful to read the very interesting scientific-social remark made by Arnon Dar at the end of the paper discussed by Guido Barbiellini at the Vulcano Workshop 2002 (Barbiellini \& Longo, 2003).

The idea that GRBs could be associated to gravitational waves (GWs) emission is now popular. Indeed, short GRBs are believed to be produced by the mergers of either double NSs or NS-BH binaries (Nakar, 2007) and the recent observation of a kilonova associated with GRB130603B (Tanvir et al., 2013; Berger, Fong \& Chornock, 2013) lends support to this hypothesis. Such compact binary coalescences generate strong GWs in the sensitive frequency band of Earth-based gravitational wave detectors (Blanchet, Iyer \& Joguet, 2002; Blanchet \& Damour, 1989). Recently, Aasi et al. (2014) searched for gravitational waves associated with 223 GRBs detected by the InterPlanetary Network (IPN) in 2005-2010 during LIGO's fifth and sixth science runs and Virgo's first, second, and third science runs. No evidence of a gravitational wave signal associated with any of the IPN GRBs in the sample, nor evidence for a population of weak gravitational wave signals associated with the GRBs has been found.

\subsection{Star Formation}

In his splendid review, Robert C. Kennicutt, Jr. (1998) discussed the observations of star formation rates (SFRs) in galaxies that provide vital clues to the physical nature of the Hubble sequence and showing that these observations are key probes of the evolutionary histories of galaxies. Kennicutt, Jr \& Evans II (2012) reviewed the progress over the previous decade in observations of large-scale star formation, with a focus on the interface between extragalactic and Galactic studies. Methods of measuring gas contents and star-formation rates have been discussed, and updated prescriptions for calculating star-formation rates were provided. They reviewed relations between star formation and gas on scales ranging from entire galaxies to individual molecular clouds.

The key dynamical processes involved in star formation - turbulence, magnetic fields, and self-gravity - are highly nonlinear and multidimensional. Therefore, it is extremely difficult a complete quantitative description of the physics involved in the process of star formation. McKee \& Ostriker (2007) attempted to review the theory of star formation. For this reason they divided star formation into large-scale and small-scale regimes and reviewed each in turn. Large scales range from galaxies to giant molecular clouds (GMCs) and their substructures. Important problems include how GMCs form and evolve, what determines the star formation rate (SFR), and what determines the initial mass function (IMF) have been discussed. Small scales range from dense cores to the protostellar systems they beget. They discussed formation of both low- and high-mass stars, including ongoing accretion. The development of winds and outflows is increasingly well understood, as are the mechanisms governing angular momentum transport in disks. However, they concluded that a comprehensive theory of star formation will be tested by the next generation of telescopes.

Fumagalli et al. (2012) investigated the evolution of the $\mathrm{H}_{\alpha}$ equivalent width, $\mathrm{EW}\left(\mathrm{H}_{\alpha}\right)$, with redshift and its dependence on stellar mass, using the first data from the 3D-HST survey, a large spectroscopic Treasury program with the HST-WFC3. Combining these data with those from ground-based telescopes, they found that at all masses the characteristic $\mathrm{EW}\left(\mathrm{H}_{\alpha}\right)$ is decreasing towards the present epoch, and that at each redshift the $\mathrm{EW}\left(\mathrm{H}_{\alpha}\right)$ is lower for high-mass galaxies. 


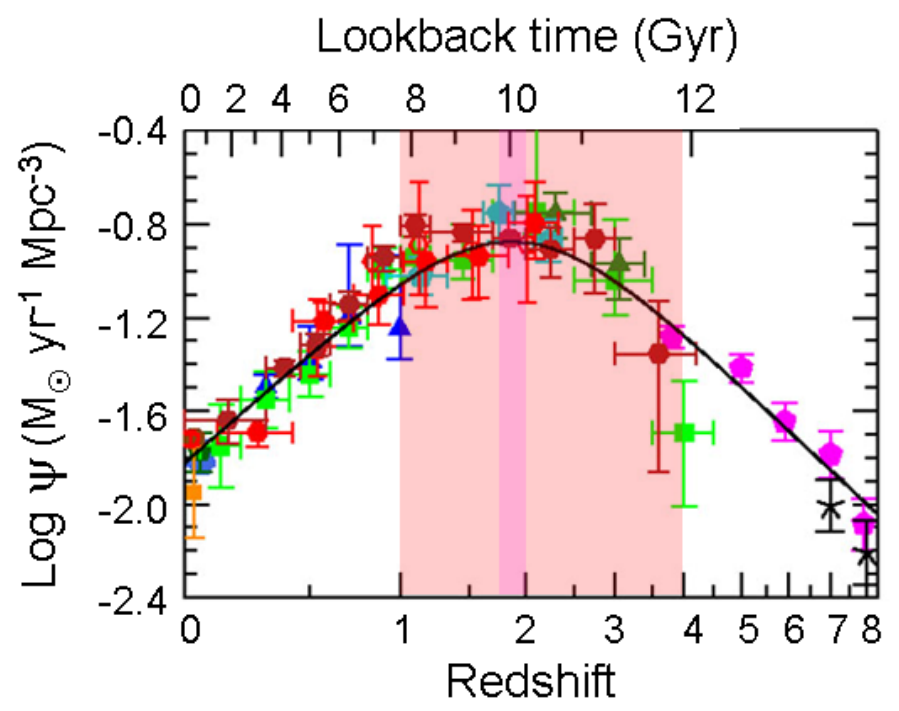

Figure 8: Evolution of SFR density with redshift (after Madau \& Dickinson, 2014).

The cosmic history of star formation, heavy element production, and reionization of the Universe from the cosmic "dark ages" to the present epoch has been discussed in the recent review paper by Madau \& Dickinson (2014). A consistent picture is emerging, whereby the star-formation rate density peaked approximately $3.5 \mathrm{Gyr}$ after the Big Bang, at $\mathrm{z} \approx 1.9$, and declined exponentially at later times, with an e-folding timescale of 3.9 Gyr. Half of the stellar mass observed today was formed before a redshift $\mathrm{z}=1.3$. About $25 \%$ formed before the peak of the cosmic star-formation rate density, and another $25 \%$ formed after $z=0.7$. Less than $\sim 1 \%$ of today's stars formed during the epoch of reionization.

However, these results were already largely discussed and presented by Hopkins \& Beacom (2006), and later by Madau \& Dickinson (2014) and summarized in the Fig. 8. The light-red rectangle marks the range of redshift where the star formation density had the maximum whose peak is at $\mathrm{z} \sim 1.9 \pm 0.1$ and marked with the light fuchsia rectangle. This will be better understood when the supernova rate density evolution, the ranges of stellar masses leading to core-collapse and type Ia supernovae, and the antineutrino and neutrino backgrounds from core-collapse supernovae will be known thanks to the next generation experiments both ground- and space-based.

\subsection{Galactic compact sources}

In the Galaxy there are different kinds of compact sources: white dwarfs (WDs), neutron stars (NSs) and black holes (BHs), both isolated and in binary systems. Thousand papers about these cosmic sources are available in the literature. We mention the last available exhaustive review by Postnov \& Yungelson (2014) about "The Evolution of Compact Binary Stars Systems in which they review the formation and evolution of compact binary stars consisting of WDs, NSs, and BHs. Merging of compact-star binaries are expected to be the most important sources for forthcoming gravitational-wave (GW) astronomy.

Several review papers have been published for discussing the different classes of galactic compact sources: i) Cataclysmic Variables (CVs) and related objects (e.g. Giovannelli \& Sabau- 
Graziati, 2008, 2012b; 2015); ii) High Mass X-ray Binaries (HMXBs) (e.g. Giovannelli \& SabauGraziati, 2001, 2004, 2014b, and van den Heuvel, 2009 and references therein; iii) Obscured Sources and Supergiant Fast X-Ray Transients (e.g. Chaty, 2011); iv) Ultra-Compact DoubleDegenerated Binaries (e.g. Wu, Ramsay \& Willes, 2008; Wu, 2009); Magnetars (Kitamoto et al., 2014: White Paper for ASTRO-H Space X-ray observatory).

A summary of these topics can be found in the review paper by Giovannelli \& Sabau-Graziati (2014a).

\subsection{Neutrinos}

One of the most important questions of fundamental physics, that is still unanswered today, is the reason for our existence, namely why the Universe is made up mostly of matter. To put it in more microscopic terms, the important unanswered question relates to a theoretical understanding of the magnitude of the observed Baryon Asymmetry in the Universe (BAU). According to the Big Bang theory, matter and antimatter have been created at equal amounts in the early Universe. The observed charge-parity (CP) violation in particle physics (Christenson, et al., 1964), prompted Sakharov (1991) to conjecture that non-equilibrium physics in the early Universe produces Baryon number (B), charge (C) and charge-parity (CP) violating, but CPT conserving, interactions/decays of anti-particles in the early Universe, resulting in the observed baryon-anti-baryon asymmetry. In fact there are two types of non-equilibrium processes in the early Universe that could produce this asymmetry: the first type concerns processes generating asymmetries between leptons and antileptons (Leptogenesis), while the second produces asymmetries between baryons and anti-baryons (Baryogenesis) (Abazajian et al., 2012).

The knowledge of the neutrino physics is fundamental for answering to this fundamental question. However, we cannot enter in a deep discussion about the physics of neutrinos. This argument deserves particular attention and space for discussion. A deep and exhaustive discussion about neutrinos can be found in the Light Sterile Neutrinos: A White Paper (Abazajian et al., 2012), Neutrino Oscillation Physics Potential of the T2K Experiment (Abe et al., 2015), and in Neutrino Physics with JUNO (An et al., 2015). However, we want to spend a few words about an important result recently obtained.

Neutrino oscillations are consistently described by three families $v_{1}, v_{2}, v_{3}$ with mass values $\mathrm{m}_{1}, \mathrm{~m}_{2}$ and $\mathrm{m}_{3}$ that are connected to the flavor eigenstates $v_{e}, v_{\mu}$ and $v_{\tau}$ by a mixing matrix $\mathrm{U}$. The neutrino oscillation probability depends on: i) three mixing angles, $\Theta_{12}, \Theta_{23}, \Theta_{13}$; ii) two mass differences, $\Delta \mathrm{m}_{12}^{2}=\mathrm{m}_{22}-\mathrm{m}_{21}, \Delta \mathrm{m}_{23}^{2}=\mathrm{m}_{23}-\mathrm{m}_{22}$; iii) and a Charge-Parity (CP) phase $\delta_{\mathrm{CP}}$. The mixing angle $\Theta_{13}$ is the key parameter of three-neutrino oscillations and regulates at the first order all the oscillation processes that could contribute to the measurement of mass hierarchy and leptonic CP violation (Mezzetto, 2011). Indeed, the neutrino mixing angle $\Theta_{13}$ is at the focus of current neutrino research. Fogli et al. (2008) reported hints in favor of $\Theta_{13}>0$ at $90 \%$ C.L.. Such hints are consistent with the recent indications of $v_{\mu} \rightarrow v_{e}$ appearance in the T2K (Abe et al., 2012) and MINOS long-baseline accelerator experiments as reported by Fogli et al. (2011). They found $\sin ^{2} \Theta_{13}=0.021 \pm 0.007$ or $\sin ^{2} \Theta_{13}=0.025 \pm 0.007$, depending on reactor neutrino flux systematics.

The evidence for $\sin ^{2} \Theta_{13}>0$ opens the door to CP violation searches in the neutrino sector, with profound implications for our understanding of the matter-antimatter asymmetry in the uni- 
verse. Fogli et al. (2012) found interesting indications for $\Theta_{23}<\pi / 4$ and possible hints for $\delta \sim \pi$, with no significant difference between normal and inverted mass hierarchy.

\subsection{Habitable Zone in the Milky Way and Exoplanets}

The most important questions about the possible origin of life in our Universe became a real scientific question in the last couple decades when it appeared a near certainty that other planets must orbit other stars. And yet, it could not be proven, until the early 1990's. Then, radio and optical astronomers detected small changes in stellar emission which revealed the presence of first a few, and now many, planetary systems around other stars. We call these planets "exoplanets" to distinguish them from our own solar system neighbors (http://science.nasa.gov/astrophysics/focusareas/exoplanet-exploration/).

Fig. 9 shows the distribution of Kepler planet candidates by size as of January 2015 (Image Credit: NASA Ames/W Stenzel). As we can see there are 808 Earth-like planets in the neighbourhood of solar system.

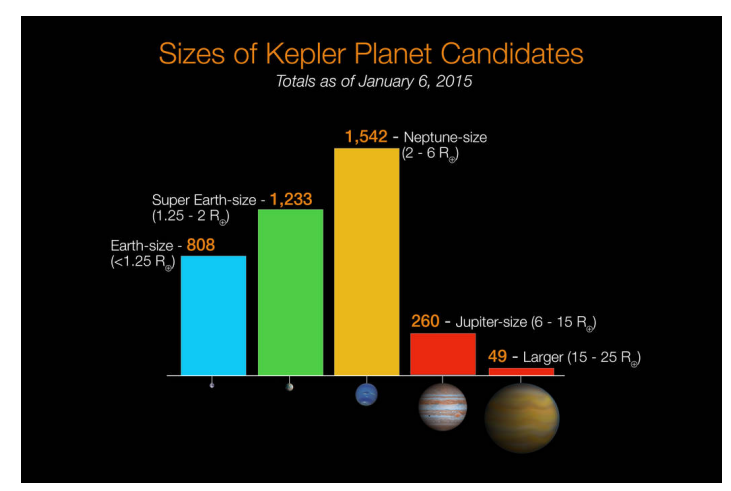

Figure 9: The distribution of Kepler planet candidates by size as of January 2015 (Image Credit: NASA Ames/W Stenzel).

The research of potential habitable exoplanets has been strongly supported during last two decades. Indeed, this field of astrophysics is now probably the most exciting since the discovery of planets Earth-like could open a serious debate about the possibility of life outside of solar system.

The list of the potential habitable exoplanets updated to 23rd July 2015 (Planetary Habitable Laboratory - PHL - University of Puerto Rico at Arecibo, http://phl.upr.edu/projects/habitableexoplanets-catalog) contains 31 objects: 10 Earth-size planets and 21 super-Earth-size planets. This list is continuously updated and the number of such exoplanets is rapidly increasing.

The presence of numerous exoplanets in the vicinity of solar system - within a distance of $\sim 0.8 \mathrm{pc}$ - plays an important role in speculating about the possible number of such exoplanets within the whole habitable zone of our galaxy. Such habitable zone has an internal radius of $\sim 4$ $\mathrm{kpc}$ and an external radius of $\sim 11 \mathrm{kpc}$, as shown in Fig. 10 (after Lineweaver, Fenner \& Gibson, 2004), where the habitable zone in a Milky Way-like galaxy is represented in green. The number of stars contained in this zone is $\approx 10 \%$ of the total number of stars in the Galaxy. Taking into account that the thickness of the disk is $\approx 1 \mathrm{kpc}$, as evaluated by the differential rotation of the Galaxy, the habitable volume is $\sim 330 \mathrm{kpc}^{3}$. Therefore, if in a volume of $\approx 2 \mathrm{pc}^{3}$ there are 808 
Earth-like planets detected, in the habitable zone of our Galaxy we could expect $\approx 133 \times 10^{6}$ Earthlike planets. It is evident that the probability of finding numerous habitable planets becomes very high. Next generation instruments ground- and space-based will provide valuable information about this intriguing problem.

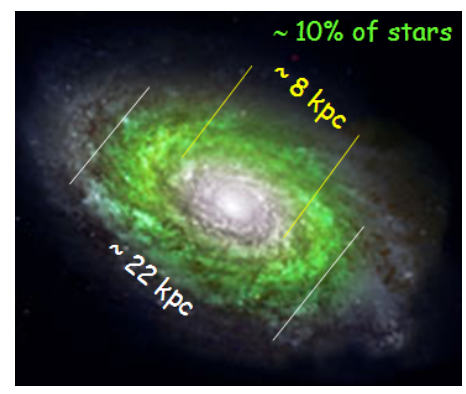

Figure 10: Habitable zone of a Milky Way-like galaxy (after Lineweaver, Fenner \& Gibson, 2004).

Studies about exoplanet predictions around stars have been performed by Bovaird \& Lineweaver (2013). They predict the existence of a low-radius $\left(R<2.5 R_{\odot}\right)$ exoplanet within the habitable zone of KOI-812 and that the average number of planets in the habitable zone of a star is 1-2.

For life-forms like us, the most important feature of Earth is its habitability. Understanding habitability and using that knowledge to locate the nearest habitable planet may be crucial for our survival as a species. During the past decade, expectations that the universe could be filled with habitable planets have been bolstered by the increasingly large overlap between terrestrial environments known to harbor life and the variety of environments on newly detected rocky exoplanets. The inhabited and uninhabited regions on Earth tell us that temperature and the presence of water are the main constraints that can be used in a habitability classification scheme for rocky planets. Lineweaver \& Chopra (2012) compiled and reviewed the recent exoplanet detections suggesting that the fraction of stars with planets is $100 \%$, and that the fraction with rocky planets may be comparably large. They reviewed extensions to the circumstellar habitable zone (HZ), including an abiogenesis habitable zone and the galactic habitable zone.

Earth is located in a dangerous part of the universe. Threats to life on Earth are manifold and range from asteroid impacts to supernova explosions and from supervolcano eruptions to humaninduced disasters. If the survival of the human species is to be ensured for the long term, then life on Earth has to spread to other planetary bodies. Mars is the most Earth-like planet we currently know and is the second closest planet; further it possesses a moderate surface gravity, an atmosphere, abundant water and carbon dioxide, together with a range of essential minerals. Thus, Mars is ideally suited to be a first colonization target. Here we argue that the most practical way that this can be accomplished is via a series of initial one-way human missions (Schulze-Makuch \& Davies, 2013).

However, we have interesting news about the presence of water in the universe. We knew that all the water found on Earth, has been transported by small bodies such as comets and asteroids. On the contrary, the work "The ancient heritage of water ice in the solar system" (Cleeves et al., 2014) has carried the knowledge one step further. It is understood that the water now present in Earth's oceans, and is present in other solar system bodies, has remained virtually unchanged with respect to that in the interstellar medium. This means that this water has not changed during the process 
of planet formation. This allows us to understand that the initial conditions that have favored the emergence of life are not unique, i.e. not dependent on the unique characteristics of our solar system. They can, however, be common in space.

An intriguing question about the probability of finding a number of civilization in the Galaxy arises. It is now evident that Drake's formula (Drake, 1962) must be object of a robust revision.

For years, the search for manifestations of extraterrestrial civilizations has been one of humanity's most ambitious projects. Major efforts are now focused on the interception of messages from extraterrestrial civilizations, and the millimeter range is promising for these purposes (Dyson,1960). The Millimetron space observatory is aimed at conducting astronomical observations to probe a broad range of objects in the Universe in the wavelength range $20 \mu \mathrm{m}$ to $20 \mathrm{~mm}$, including the search for extraterrestrial life (Kardashev et al., 2014, and the references therein).

However, if we insist in looking for life which is like our own, why do we look for ... INTELLIGENT LIFE? (Giovannelli, 2001b).

\subsection{Prospects for the Astrophysics of Next Decades: the Contribution of Small Experiments}

It is evident that the future of the research in astrophysics must take into account the three main methods to tackle the way of the knowledge of our Universe: photonic astrophysics, neutrino astrophysics, and particle astrophysics. The synergy of these methods converge in what we call now astroparticle physics.

With the sensitivity of the Cherenkov Telescope Array (CTA) we expect the detection of thousands VHE $\gamma$-ray sources. With the advent of the European-Extreme Large Telescope: E-ELT we will be witnesses of astonishing results. E-ELT is considered worldwide as one of the highest priorities in ground-based astronomy. It will vastly advance astrophysical knowledge in optical and near infrared (NIR) regions, allowing detailed studies of subjects including planets around other stars, the first objects in the Universe, super-massive black holes, and the nature and distribution of the dark matter and dark energy which dominate the Universe. GAIA (e.g. Rix \& Bovy, 2013), the new generation astrometric satellite after HIPPARCOS, already in flight, will provide strong impact on stellar evolution and in calibrating the energetic of cosmic sources thanks to $\sim 10 \%$ accuracy in determing the stellar distances and stellar velocities with resolution of $\sim 1 \mathrm{~km} \mathrm{~s}^{-1}$. Another important jump in the knowledge of our Universe will come from the James Webb Space Telescope (JWST) that will be launched in orbit in 2018. The JWST will be a giant leap forward in our quest to understand the Universe and our origins. JWST will examine every phase of cosmic history, namely the Bridge between the Big Bang and Biology: from the first luminous glows after the Big Bang to the formation of galaxies, stars, and planets to the evolution of our own solar system. Moreover, JWST will tell us more about the atmospheres of exoplanets, and perhaps even find the building blocks of life elsewhere in the universe (see http://jwst.nasa.gov/science.html).

Together with such impressive big experiments it is necessary to mention the extreme importance of small experiments that constitute useful and indispensable tools for a huge number of investigations not possible with the larger ones for a great number of reasons. They can be: i) space-based experiments: small-, mini-, micro- and nano-satellites; ii) ground-based experiments: small telescopes and robotic telescopes.

An interesting example of a micro-satellite developed by the students of Tokyo Institute of Technology is TSUBAME (launched from Russia on Nov 6, 2014) for measuring hard X-ray po- 
larization of GRBs in order to reveal the nature of their central engine (Kurita et al., 2015). Other example about the complementarity of small mission to big experiments is TESS (The Transiting Exoplanet Survey Satellite). It will search for planets transiting bright and nearby stars. TESS is a next logical step after NASAŠs Kepler mission that revolutionized exoplanetary science by revealing that planets with sizes between those of the Earth and Neptune are abundant (Borucki et al., 2011; Fressin et al., 2013). TESS has been selected by NASA for launch in 2017 as an Astrophysics Explorer mission. The longest observing intervals will be for stars near the ecliptic poles, which are the optimal locations for follow-up observations with the JWST. TESS is expected to find more than a thousand planets smaller than Neptune, including dozens that are comparable in size to the Earth (Ricker et al., 2015). One example more is the study of a small satellite mission HiZ-GUNDAM for future observations of GRBs. The mission concept is to probe "the end of dark ages and the dawn of formation of astronomical objects", i.e. the physical condition of early universe beyond the redshift $\mathrm{z}>7$ (Yonetoku et al., 2014). Small space missions, complementary to those Earth-based, even larger, are also devoted to investigate cosmic rays at very high energies, like for instance the NUCLEON space experiment designed to investigate directly, above the atmosphere, the energy spectra of cosmic-ray nuclei and the chemical composition $(Z=1-30)$ at energy range $100 \mathrm{GeV}-1000 \mathrm{TeV}$, including the "knee" energy range (Atkin et al., 2015). NUCLEON experiment on board of the RESURS-P satellite was launched on December 26, 2014.

Probably the best example of the importance of small missions is the joint JAXA/NASA ASTRO-H mission, the sixth in a series of highly successful X-ray missions developed by the Institute of Space and Astronautical Science (ISAS) (e.g. Takahashi et al., 2014a). The launch date was decided to be on February 12, 2016. ASTRO-H is expected to provide breakthrough results in scientific areas as diverse as the large-scale structure of the Universe and its evolution, the behaviour of matter in the gravitational strong field regime, the physical conditions in sites of cosmic-ray acceleration, and the distribution of dark matter in galaxy clusters at different redshifts (Takahashi et al., 2014b - ASTRO-H Space X-ray Observatory: White Paper - in which the series of white papers, dedicated to the different topics, is listed).

As we have discussed in section 3.4, how reionization occurred is still an open problem that deserves particular attention. Indeed, WISH (Wide-field Imaging Surveyor for High-redshifts) is a space mission concept to conduct very deep and widefield surveys at NIR wavelength at 1-5 $\mu \mathrm{m}$ to study the properties of galaxies at very high redshift beyond the epoch of cosmic reionization (Yamada et al., 2012).

It is important to remark the contribution to science provided by: i) Robotic Autonomous Observatories (Castro-Tirado, 2010); ii) Global Robotic Telescopes Intelligent Array for E-Science (GLORIA) (Castro-Tirado et al., 2014, 2015); iii) (B)urst (O)bserver and (O)ptical (T)ransient (E)xploring (S)ystem (BOOTES) (Castro-Tirado et al., 2012). With the installation of the fifth telescope of the BOOTES worldwide network of robotic telescopes it is possible a continuous monitoring of astronomical targets (Hiriart, 2014); iv) Pi of the Sky (a system of robotic telescopes designed for observations of short timescale astrophysical phenomena, e.g. prompt optical GRB emissions) (Siudek et al., 2011). PI of the Sky is now part of the GLORIA system (Mankiewicz et al., 2014; Obara et al., 2014); v) MITSuME(Multicolor Imaging Telescope for Survey and Monstrous Explosions). MITSuME telescopes were designed to perform "real time" and "automatic" follow-up observations for gamma-ray bursts (GRBs) prompted by the GCN alerts via the internet 
(Shimokawabe et al., 2008). An example of follow-up observations for GRBs and black hole binary is briefly reported by Saito et al. (2012, 2014); vi) CHASE (CHilean Automatic Supernova sEarch) a project began in 2007 with the goal to discover young, nearby southern supernovae in order to: i) better understand the physics of exploding stars and their progenitors; ii) refine the methods to derive extragalactic distances. During the first four years of operation, CHASE has produced more than 130 supernovae (Hamuy et al., 2012); vii) MUSICOS (MUlti-SIte COntinuous Spectroscopy) project, whose purpose is to organize multisite continuous spectroscopic observations (Baudrand \& Böhm, 1992; Foing et al., 1992); viii) WET (Whole Earth Telescope) (Nather et al., 1990). The idea born in 1986 when scientists from the University of Texas Astronomy Department established a world-wide network of cooperating astronomical observatories to obtain uninterrupted time-series measurements of variable stars. This approach has been extremely successful, and has placed the fledgling science of stellar seismology at the forefront of stellar astrophysics (e.g. Handler et al., 1996; Kilkenny et al., 2003; Reed et al., 2011); ix) MASTER (Mobile Astronomical System of the TElescope-Robots). The main goal of the MASTER-Net project is to produce a unique fast sky survey with all sky observed over a single night down to a limiting magnitude of 19-20. Such a survey will make it possible to address a number of fundamental problems: search for dark energy via the discovery and photometry of supernovae (including SNIa), search for exoplanets, microlensing effects, discovery of minor bodies in the Solar System, and space-junk monitoring. All MASTER telescopes can be guided by alerts, and we realize the observations of prompt optical emission from GRBs synchronously in several filters and in several polarization planes (Lipunov et al., 2010; see also http://observ.pereplet.ru/MASTER(underscore)OT.html). With the addition of the SAAO Telescope "farm" on the Sutherland plateau, MASTER-SAAO is giving a massive contribution to transient alerts in AstronomerŠs telegrams for different classes of cosmic sources, such as GRBs, SNe, Blazars, Novae, X-ray pulsars, CVs, fast rapid transient etc. (e.g at August 2015, 530 new CVs have been discovered) (Buckley, 2015).

\section{Conclusions}

After this discussion it appears evident the importance of multi-frequency astrophysics from ground- and space-based experiments in order to deeply explore the nature of our Universe. However, there are many problems in performing simultaneous multi-frequency, multi-site, multiinstrument, multi-platform measurements due to: i) objective technological difficulties; ii) sharing common scientific objectives; iii) problems of scheduling and budgets; iv) politic management of science.

We hope to have given enough arguments in favor of small experiments, of course not rejecting the extremely importance of large experiments, especially those looking at the first stages of the life of our Universe. As example of small space-based experiments dedicated to specific investigations, we have mentioned TSUBAME for measuring hard X-ray polarization of GRBs, TESS for searching planets transiting bright and nearby stars, HiZ-GUNDAM for probing the end of dark ages and the dawn of formation of astronomical objects, WISH for studying the properties of galaxies at very high redshift beyond the epoch of cosmic reionization, NUCLEON for investigating directly the energy spectra of cosmic-ray nuclei and their chemical composition, and ASTRO-H, that, in spite of its small size, will provide breakthrough results in many different scientific areas. ${ }^{(*)}$ 
And for the ground-based small experiments we have mentioned the small telescopes (SmTs) - including those belong to amateurs - that are the unique capable of doing long-term observations of selected sources, usually forbidden with larger telescopes. SmTs - spread along the longitude and grouped in specific programs (e.g. WET, MUSICOS, BOOTES, GLORIA, MASTER) - can provide continuous long-term monitor of selected sources without night-day interruption (i.e. sdB stars for stellar seismology, RS CVn stars, XRBs, CVs, GRBs, survey of asteroids,...). Thus, SmTs - better if robotic - are unreplaceable tools complementary to larger telescopes and to ground- and space-based multi-frequency experiments.

(*) Unfortunately, during the process of editing this review, JAXA (Japan Aerospace Exploration Agency) gave on March 27, 2016 (JST) a communication anomaly of X-ray Astronomy Satellite "Hitomi" (ASTRO-H): The Japan Aerospace Exploration Agency (JAXA) found that communication with the X-ray Astronomy Satellite "Hitomi" (ASTRO-H), launched on February 17, 2016 (JST), failed from the start of its operation originally scheduled at 16:40, Saturday March 26 (JST). Up to now, JAXA has not been able to figure out the state of health of the satellite.

Acknowledgments This research has made use of NASA's Astrophysics Data System.

\section{References}

[1] Aaij, R. et al., 2015, arXiv:1507.03414v2 [hep-ex] 25 Jul

[2] Aasi, J. et al., 2014, PhRvL 113, 011102

[3] Abazajian, K.N. et al., 2012, arXiv:1204.5379v1 [hep-ph] 18 Apr 2012

[4] Abe, K, et al., 2012, Nucl. Instr. \& Meth. in Phys. Res. A, 659, 106

[5] Abe, K, et al., 2015, arXiv:1409.7469v2 [hep-ex] 10 Feb 2015

[6] Abdo, A.A. et al.: 2010, ApJ, 723, 1082

[7] Ade, P.A.R. et al. (BICEP Collaboration), 2015, PhRvL, 114, 1301

[8] Albert, J. \& Magic Collaboration: 2008, Science, 320, 1752

[9] An, F. et al., 2015, arXiv:1507.05613v2 [physics.ins-det] 18 Oct 2015

[10] Anders, M. et al., 2013, EPJA, 49, 28.

[11] Anders, M. et al., 2014, PhRvL 113d2501A.

[12] Atkin, E. et al., 2015, EPJ Web of Conferences 105, 01002

[13] Barbiellini, G. \& Longo,F., 2003, in Frontier Objects in Astrophysics and Particle Physics, F. Giovannelli \& G. Mannocchi (Eds.), SIF, Bologna, Italy, 85, 281

[14] Bartelmann, M., 2008, in Rev. Mod. Astron. (Siegfried Röser Ed.), Vol. 20: Cosmic Matter, p. 92.

[15] Baudrand, J \& Böhm, T., 1992, A\&A, 259, 711

[16] Beall, J.H.: 2002, in Multifrequency Behaviour of High Energy Cosmic Sources, F. Giovannelli \& L. Sabau-Graziati (Eds.), Mem. S.A.It., 73, 379. 
[17] Beall, J.H.: 2003, in Multifrequency Behaviour of High Energy Cosmic Sources, F. Giovannelli \& L. Sabau-Graziati (Eds.), ChJA\&A Suppl., 3, 373.

[18] Beall, J.H.: 2014a, in Multifrequency Behaviour of High Energy Cosmic Sources - X, F. Giovannelli \& L. Sabau-Graziati (Eds.), Acta Polytechnica CTU Proceedings 1(1), 259-264.

[19] Beall, J.H.: 2014b, in Multifrequency Behaviour of High Energy Cosmic Sources - XI, F. Giovannelli \& L. Sabau-Graziati (Eds.), http://pos.sissa.it/cgi-bin/reader/conf.cgi?confid=237

[20] Beall, J.H., Guillory, J., Rose, D.V., 1999, in Multifrequency Behaviour of High Energy Cosmic Sources, F. Giovannelli \& L. Sabau-Graziati (Eds.), Mem. S.A.It., 70, 1235.

[21] Beall, J.H. et al.: 2006, in Multifrequency Behaviour of High Energy Cosmic Sources, F. Giovannelli \& L. Sabau-Graziati (Eds.), ChJA\&A Suppl., 6 Suppl. 1, 283.

[22] Beall, J.H. et al.: 2007, in Frontier Objects in Astrophysics and Particle Physics, F. Giovannelli \& G. Mannocchi (Eds.), SIF, Bologna, Italy, 93, 315.

[23] Beatty, J.J. et al., 2013, arXiv:1310.5662v2

[24] Bednarek, W. et al.: 1990, A\&A 236, 268

[25] Begelman, M.C., Blandford, R.D. \& Rees, M.J., 1984, Rev. Mod. Phys., 56, 255

[26] Bennett, C.L. et al., 2013, ApjS, 208, 20

[27] Bennett, C.L. et al., 2014, ApJ, 794, 135

[28] Berger, E., 2014, Annu. Rev. A\&A, 52, 43

[29] Berger, E., Fong, W. \& Chornock, R., 2013, ApJL 774, L23

[30] de Bernardis et al., 2000, Nature, 404, 955

[31] Blanchet, L., Iyer, B.R. \& Joguet, B., 2002, Phys. Rev. D 65, 064005

[32] Blanchet, L. \& Damour, T., 1989, Annales Inst. H. Poincaré Phys. Théor. 50, 377

[33] Borucki et al., 2011, ApJ, 736, 19

[34] Bovaird, T. \& Lineweaver, C.H., 2013, MNRAS 435, 1126

[35] Buckley, D., 2015, talk at the Palermo Workshop on "The Golden Age of Cataclysmic Variables and Related Objects - III"

[36] Burles, S. et al., 2001, ApJ, 552, L1.

[37] Buson, S., 2014, in Frobtier Research in Astrophysics, Franco Giovannelli \& Lola Sabau-Graziati (eds.), http://pos.sissa.it/cgi-bin/reader/conf.cgi?confid=237

[38] Cardini, A., 2015, Interview of Alessandro Cardini, P.I. of LHCb, 14th July 2015

[39] Castro-Tirado, A.J. et al., 1994, ApJSS, 92, 469

[40] Castro-Tirado, A.J., 2010, Hindawi Publ. Co. Adv. in Astron., Vol. 2010, ID 570489, 8 pages

[41] Castro-Tirado, A.J., 2012, in Second Workshop on Robotic Autonomous Observatories, Sergey Guziy et al. (eds.), ASI Conf. Ser., 7, 313

[42] Castro-Tirado, A.J. et al., 2014, in Robotic Astronomy 2013, J.C. Tello et al. (eds.), Rev. Mex. A\&A Conf. Ser., 45, 104 
[43] Castro-Tirado, A.J., 2015, in Highlights of Spanish Astrophysics VIII, Proc. of the XI Scientific Meeting of the Spanish Astronomical Society, A.J. Cenarro et al. (eds.), p. 895

[44] Chaty, S., 1998, Ph.D. thesis, University Paris XI

[45] Chaty, S., 2011, in Evolution of Compact Binaries, L. Schmidtobreick, M. Schreiber \& C. Tappert (Eds.), ASP Conf. Ser., 447, 29

[46] Christenson, J.H et al., 1964, PhRvL, 13, 138

[47] Clarke, D.A. et al.: 2008, Physics in Canada, 64(2), 48

[48] Cleeves L. Ilsedore et al., 2014, Science, 345, 1590

[49] Coppi, P.S. \& Aharonian, F.A., 1997, ApJ, 487, L9

[50] Costamante, L., 2012, in Multifrequency Behaviour of High Energy Cosmic Sources, F. Giovannelli \& L. Sabau-Graziati (Eds.), Mem. SAIt., 83, 138

[51] Crites, A.T. et al., 2015, ApJ, 805, 36

[52] Cucchiara, A. et al.: 2011, ApJ, 736, 7

[53] Damon, E. et al., 2004, Measurements of the Hubble Constant, D.B. Campbell \& J. Deneva (eds.), Proc. of the Fall 2004 Astronomy 233 Symposium, Cornell University Astronomy Department and the College of Arts and Sciences under the John S. Knight Institute Sophomore Seminar Program, pp. $1-61$

[54] Di Leva, A. et al., 2014, PhRv C, 89, Issue 1, id.015803 and erratum PhRv C, 90, Issue 1, id.019902

[55] Domínguez, A. et al., 2011, MNRAS, 410, 2556

[56] Dopita, M.A.: 2011, Astrophys. Space Sci., 335, 345

[57] Dyson, F., 1960, Science, 131, 1667

[58] Drake, F.D.: 1962, Intelligent Life in Space, New York: Macmillan, 128 pp.

[59] Fan, X. et al.: 2003, AJ 125, 1649

[60] Fogli, G.L. et al., 2008, Ph. Rev. Letter, 101, 141801

[61] Fogli, G.L. et al., 2011, Phys. Rev. D, 84, 053007

[62] Fogli, G.L. et al., 2012, Phys. Rev. D, 86, 013012

[63] Foing, B.H. et al., 1992, in Cool stars, stellar systems, and the sun, Proc. of the 7th Cambridge Workshop, ASP Conf. Ser., 26, 637

[64] Fressin, F. et al., 2013, ApJ, 766, 81

[65] Fumagalli, M. et al.: 2012, APJL, 757, L22

[66] Gell-Mann, M., 1964, PhL, 8, 214

[67] Giallongo, E. et al.: 1994, ApJ, 425, L1

[68] Gianotti, F., 2012, 4th July-talk at CERN

[69] Giovannelli, F. (ed.), 2001a, The Bridge between the Big Bang and Biology (Stars, Planetary Systems, Atmospheres, Volcanoes: Their Link to Life), President Bureau of the CNR, Roma, Italy, pp. 1-440 
[70] Giovannelli, F., 2001b, in The Bridge between the Big Bang and Biology (Stars, Planetary Systems, Atmospheres, Volcanoes: Their Link to Life), F. Giovannelli (ed.), President Bureau of the CNR, Roma, Italy, p. 439

[71] Giovannelli, F., 2013, Acta Polytechnica 53(Suppl.), 483

[72] Giovannelli, F. \& Polcaro, V.F.: 1986, MNRAS, 222, 619-627 (GP86)

[73] Giovannelli, F. \& Sabau-Graziati, L., 2001, Ap\&SS, 276, 67

[74] Giovannelli, F., Sabau-Graziati, L., 2004, SSR, 112, 1

[75] Giovannelli, F. \& Sabau-Graziati, L., 2008, Chinese J. A\&A Suppl., 8, 1

[76] Giovannelli, F., Sabau-Graziati, L., 2012a, in Multifrequency Behaviour of High Energy Cosmic Sources, Franco Giovannelli \& Lola Sabau-Graziati (eds.), Mem. SAIt. 83, 17

[77] Giovannelli, F. \& Sabau-Graziati, L., 2012b, in The Golden Age of Cataclysmic Variables and Related Objects, F. Giovannelli \& L. Sabau-Graziati (Eds.), Mem. SAIt., 83 N. 2, 440

[78] Giovannelli, F., Sabau-Graziati, L., 2014a, in Frontier Research in Astrophysics, Franco Giovannelli \& Lola Sabau-Graziati (eds.), http://pos.sissa.it/cgi-bin/reader/conf.cgi?confid=237

[79] Giovannelli, F. \& Sabau-Graziati, L., 2014b, Acta Polytechnica CTU Proc., 1(1), 1

[80] Giovannelli, F. \& Sabau-Graziati, L., 2015, Acta Polytechnica CTU Proc., 2, 3

[81] Gnedin, N.Y., 2000, ApJ, 535, 530-554

[82] de Gouveia dal Pino, E.M., 2005, Adv. in Space Res., 35, 908

[83] de Gouveia Dal Pino, E.M., Piovezan, P.P. \& Kadowaki, L.H.S., 2010, A\&A, 518, A5

[84] Granot, J., 2007, Rev.Mex, A\&A (Serie de Conferencias), 27, 140-165

[85] Granot, J., 2009, arXiv:0905.2206v1 [astro-ph.HE] 13 May 2009

[86] Granot, J. \& Ramirez-Ruiz, E., 2010, arXiv:1012.5101v1 [astro-ph.HE] 22 Dec 2010

[87] Greiner, J. et al., 2009, ApJ, 693, 1610

[88] Gustavino, C.: 2007, in Frontier Objects in Astrophysics and Particle Physics, F. Giovannelli \& G. Mannocchi (eds.), Italian Physical Society, Ed. Compositori, Bologna, Italy, 93, 191.

[89] Gustavino, C.: 2009, in Frontier Objects in Astrophysics and Particle Physics, F. Giovannelli \& G. Mannocchi (eds.), Italian Physical Society, Ed. Compositori, Bologna, Italy, 98, 77.

[90] Gustavino, C.: 2011, in Frontier Objects in Astrophysics and Particle Physics, F. Giovannelli \& G. Mannocchi (eds.), Italian Physical Society, Ed. Compositori, Bologna, Italy, 103, 657.

[91] Gustavino, C., 2012, in Nuclei in the Cosmos (NIC XII), http://pos.sissa.it/cgi-bin/reader/conf.cgi?confid=146, id.74

[92] Gustavino, C., 2013, AcPol, 53, 534

[93] Hamuy, M. et al., 2012, in Multifrequency Behaviour of High Energy Cosmic Sources, Franco Giovannelli \& Lola Sabau-Graziati (eds.), Mem. SAIt., 83, 388

[94] Handler, G. et al., 1996, A\&A, 307, 529

[95] Henry, R.C., 1999, ApJL, 516, L49 
[96] Henry, R.C.: 2002, in Multifrequency Behaviour of High Energy Cosmic Sources, Franco Giovannelli \& Lola Sabau-Graziati (eds.), Mem. SAIt., 73 N. 1, 67

[97] van den Heuvel, E.P.J.: 2009, Ap\&SS Library, 359, 125

[98] Hiriart, D., 2014, Rev. Mex. A\&A Conf. Ser., 45, 87

[99] Hopkins, A,M. \& Beacom, J.F., 2006, ApJ, 651, 142

[100] Incandela, J., 2012, UCSB/CERN, Talk on July 4, 2012

[101] Inoue, S. et al.: 2013, Astroparticle Physics 43, 252

[102] Inoue, Y. et al., 2013, ApJ, 768, 197

[103] Izzo, L. et al., 2010, J. Korean Phys. Soc., 57, No. 3, 551

[104] Kann, D.A. et al.: 2010, ApJ, 720, 1513

[105] Kaplinghat, M. et al., 2003, ApJ, 583, 24

[106] Kardashev, N.S. et al., 2014, Phys. Uspekhi, 57 (12), 1199

[107] Kennicutt, Jr., R.C., 1998, ARA\&A, 36, 189

[108] Kennicutt, Jr., R.C. \& Evans II, N.J.: 2012, ARA\&A, 50, 531

[109] Kilkenny, D. et al., 2003, MNRAS, 345, 834

[110] Kitamoto, S. et al., 2014, arXiv:1412.1165v1 [astro-ph.HE] 3 Dec 2014

[111] Klebesadel, R.W., Strong, I.B., Olson, R.A., 1973, ApJL, 182, L85

[112] Knox, L.: 2003, New Astron. Rev., 47, 883

[113] Kogut, A. et al., 2003, ApJS, 148, 161

[114] Komatsu, E. et al., 2011, ApJS, 192, 18

[115] Komatsu, E., Bennett, C.L. (on behalf of the WMAP science team), 2014, Prog. Theor. Exp. Phys., $06 \mathrm{~B} 102$

[116] Kouveliotou, C. et al., 1993, ApJ, 413, L101

[117] Krennrich, F. \& Orr, M., 2013, arXiv:1304.8057

[118] Kurita S. et al., 2015, arXiv:1503.01975

[119] Lineweaver, C.H., Fenner, Y. \& Gibson, B.K., 2004, Nature, 303, 59.

[120] Lineweaver, C.H. \& Chopra, A., 2012, Ann. Rev. of Earth and Planetary Sci., 40 (issue 1), 597

[121] Lipunov, V. et al., 2010, Hindawi Publ. Co. Adv. in Astron. Vol. 2010, ID 349171, 6 pages

[122] Loeb, A. \& Barkana, R., 2001, Annu. Rev. A\&A, 39, 19

[123] Madau, P. \& Dickinson, M., 2014, ARA\&A, 52, 415

[124] Mankiewicz, L. et al., 2014, Rev. Mex. A\&A Conf. Ser., 45, 7

[125] Marscher, A.P., 2005, Mem. S.A.It., 76, 13

[126] Mather, J.C. et al., 1990, ApJL, 354, 37

[127] Mazets, E.P., Golenetskii, S.V.: 1988, Sov. Sci. Rev. E. Astrophys. Space Phys., 6, 283 
[128] McKee, C.F. \& and Ostriker, E.C.: 2007, ARA\&A, 45, 565

[129] Meszaros, P., 2006, Rep. Prog. Phys. 69, 2259

[130] Meyer, M. et al., 2012, A\&A 542, A59

[131] Mezzetto, M., 2011, in Symposium on Prospects in the Physics of Discrete Symmetries, Journal of Physics: Conference Series 335, 012005

[132] Mineo, S. et al., 2014, MNRAS, 437, 1698

[133] Mirabel, I.F. et al., 1992, Nature, 358, 215

[134] Mirabel, I.F., Rodríguez, L.F., 1994, Nature, 371, 46

[135] Mirabel, I.F. et al., 2011, A\&A 528, A149

[136] Mirabel, I.F. et al., 2015, in Extragalactic jets from every angle, Proc. IAU Symp. No. 313, F. Massaro, C.C. Cheung, E. Lopez \& A. Siemiginowska (Eds.), pp. 370-373

[137] Nakar, E., 2007, Phys. Rep. 442, 166

[138] Nather, R.E. et al., 1990, ApJ, 361, 309

[139] Obara, L. et al., 2014, Rev. Mex. A\&A Conf. Ser., 45, 118

[140] Padovani, P., 1999, in Frontier Objects in Astrophysics and Particle Physics, F. Giovannelli \& G. Mannocchi (eds.), SIF, Vol. 65, 159

[141] Panagia, N. et al., 2005, ApJ, 633, L1

[142] Perley, D.A. et al., 2014, ApJ, 781, 37

[143] Piran, T., 2004, Rev. Mod. Phys., 76, 1143

[144] Postnov, K.A. \& Yungelson, L.R., 2014, Living Rev. Relativity, 17, 3

[145] Ramaty, R, \& Lingenfelter, R.E., 1982, Ann. Rev. Nucl. Part. Sci., 32, 235

[146] Reed, M.D. et al., 2011, MNRAS, 412, 371

[147] Ressel, M.T., Turner, M.S.: 1990, Comm. Astrophys. 14, 323

[148] Ricker, G.R. et al., 2015, Journal of Astronomical Telescopes, Instruments, and Systems 1(1), 014003

[149] Riess, A.G. et al.: 2004, ApJ, 607, 665

[150] Riess, A.G. et al., 2011, ApJ, 730, 119

[151] Rix, H-W. \& Bovy, J., 2013, A\&A Rev., 21, 61

[152] Sakharov, A.D., 1991, Usp. Fiz. Nauk, 161, 110

[153] Saito, Y. et al., 2012, in Death of Massive Stars: Supernovae and Gamma-Ray Bursts, P. Roming, N. Kawai \& E. Pian (eds.), Proc. IAU Symp. No. 279, 387

[154] Saito, Y. et al., 2014, in Suzaku-MAXI 2014: Expanding the Frontiers of the X-ray Universe, M. Ishida, R. Petre \& K. Mitsuda (eds.), Proc. Conf. held 19-22 February, 2014 at Ehime University, Japan, p. 210

[155] Schuecker, P., 2005, In Rev. Mod. Astron. (Siegfried Röser Ed.), Vol. 18, 76-105.

[156] Schuecker, P. et al., 2003, A\&A, 402, 53 
[157] Schulze-Makuch, D. \& Davies, P., 2013, JBIS, 66, 11

[158] Scott, D.A. et al., 2012, PhRvL, 109, Issue 20, id. 202501

[159] Shimokawabe, T. et al., 2008, AIPC, 1000, 543

[160] Siudek, M. et al., 2011, Acta Polytechnica, 51 No. 6, 64

[161] Spergel, D.N. et al., 2003, ApJS, 148, 175

[162] Srianand, R., Petitjean, P. \& Ledoux, C., 2000, Nature, 408, 931

[163] Straniero, O. et al., 2013, ApJ, 763, 100

[164] Strieder, F. et al., 2012, PhL B, 707, Issue 1, 60

[165] Sunyaev, R.A. \& Zeldovich, Ya.B., 1980, MNRAS, 190, 413

[166] Takahashi, T. et al., 2014a, in Space Telescopes and Instrumentation 2014: Ultraviolet to Gamma Ray, Tadayuki Takahashi et al. (eds.), Proc. SPIE, 9144, 25

[167] Takahashi, T. et al., 2014b, arXiv:1412.2351v1 [astro-ph.HE] 7 Dec 2014

[168] Tanvir, N.R. et al., 2009, Nature, 461, 1254

[169] Tanvir, N.R. et al., 2013, Nature, 500, 547

[170] Tonry, J.L. et al.: 2003, ApJ, 594, 1-24.

[171] Urry, C.M. \& Padovani, P., 1995, PASP, 107, 803

[172] Wang, F.Y., Dai, Z.G., 2009, MNRAS, 400, 10

[173] Woosley, S.E. \& Bloom, J.S., 2006, Annu. Rev. A\&A, 44, 507

[174] Wu, K., 2009, Res. Astron. Astrophys., 9 (Issue 7), 725

[175] Wu, K., Ramsay, G. \& Willes, A., 2008, Chin. J. A\&A, Vol. 8, Suppl., 169

[176] Wu, Xiangping: 2008, Talk at the Summer School on "Cosmic Reionization" at the KIAA- PKU, Beijing, China, July 1-11

[177] Yamada, T. et al., 2012, in Space Telescopes and Instrumentation 2012: Optical, Infrared, and Millimeter Wave, Proc. of the SPIE, Vol. 8442, article id. 84421A, 12 pp.

[178] Yonetoku, D. et al., 2014, Proc. of the SPIE, Vol. 9144, id. 91442S

[179] Zaldarriaga, M., 1997, Phys. Rev. D, 55, 1822

[180] Zhang, B. et al., 2013, talk at the "Multi-Messenger Transient Workshop, KIAA, China 